\title{
SPATIAL DISCRETIZATION OF RESTRICTED GROUP $C^{*}$-ALGEBRAS
}

\author{
STEFFEN ROCH
}

Abstract. We consider spatial discretizations by the finite sections method of the restricted group $C^{*}$-algebra of a finitely generated discrete group, which is represented as a concrete operator algebra via its left-regular representation. Special emphasis is paid to the quasicommutator ideal of the algebra generated by the finite sections sequences and to the stability of sequences in that algebra. For both problems, the sequence of the discrete boundaries plays an essential role. Finally, for commutative groups and for free non-commutative groups, the algebras of the finite sections sequences are shown to be fractal.

Mathematics subject classification (2010): 46L99, 47N40, 47L80, 65J10.

Keywords and phrases: Restricted group $C^{*}$-algebras, finite sections, discrete boundaries, stable sequences, fractal algebras.

\section{REFERENCES}

[1] T. ADACHI, A note on the Folner condition for amenability, Nagoya Math. J., 131 (1993), 67-74.

[2] B. BlackAdAR, Operator Algebras. Theory of $C^{*}$-Algebras and von Neumann Algebras, Springer, Berlin, Heidelberg 2006.

[3] A. Böttcher, S. M. Grudsky, Spectral Properties of Banded Toeplitz Matrices, Siam, Philadelphia 2005.

[4] A. Böttcher, B. Silbermann, Introduction to Large Truncated Toeplitz Matrices, SpringerVerlag, Berlin, Heidelberg 1999.

[5] N. P. Brown, N. Ozawa, $C^{*}$-Algebras and Finite-Dimensional Approximations, Graduate Studies Math. 88, Amer. Math. Soc., Providence, R. I., 2008.

[6] L. A. Coburn, The $C^{*}$-algebra generated by an isometry, Bull. Am. Math. Soc., 73 (1967), $722-726$.

[7] K. R. Davidson, $C^{*}$-Algebras by Example, Fields Institute Monographs Vol. 6, Providence, R. I., 1996.

[8] R. Hagen, S. Roch, B. Silbermann, Spectral Theory of Approximation Methods for Convolution Equations, Birkhäuser Verlag, Basel, Boston, Berlin 1995.

[9] R. Hagen, S. Roch, B. Silbermann, $C^{*}$-Algebras and Numerical Analysis, Marcel Dekker, Inc., New York, Basel 2001.

[10] M. Lindner, Stable subsequences of the finite section method, J. Appl. Numer. Math., 60 (2010), 501-512.

[11] V. S. RABINOVICH, S. RoCH, Fredholm properties of band-dominated operators on periodic discrete structures, to appear in Complex Anal. Oper. Theory.

[12] V. S. Rabinovich, S. Roch, B. Silbermann, Fredholm theory and finite section method for band-dominated operators, Integral Equations Oper. Theory 30 (1998), 452-495.

[13] V. S. RAbinovich, S. Roch, B. Silbermann, Band-dominated operators with operator-valued coefficients, their Fredholm properties and finite sections, Integral Eq. Oper. Theory, 40, 3 (2001), 342-381.

[14] V. S. Rabinovich, S. Roch, B. Silbermann, Limit Operators and their Applications in Operator Theory, Oper. Theory: Adv. Appl. 150, Birkhäuser, Basel 2004.

[15] V. S. Rabinovich, S. Roch, B. Silbermann, On finite sections of band-dominated operators, in: Operator Algebras, Operator Theory and Applications (Eds. M. A. Bastos, I. Gohberg, A. B. Lebre, F.-O. Speck), Oper. Th.: Adv. Appl. 181, Birkhäuser, Basel, Boston, Berlin 2008. 
[16] M. Reed, B. Simon, Methods of Modern Mathematical Physics. Volume 1: Functional Analysis, Academic Press, New York, London 1972.

[17] S. Roch, Algebras of approximation sequences: Fractality, Oper. Theory: Adv. Appl. 121, Birkhäuser, Basel 2001, 471-497.

[18] S. RocH, Finite sections of band-dominated operators, Memoirs AMS Vol. 191, 895, Providence, R.I., 2008.

[19] S. Roch, Spatial discretization of Cuntz algebras, Houston Math. J., to appear.

[20] S. Roch, B. Silbermann, $C^{*}$-algebra techniques in numerical analysis, J. Oper. Theory, 35, 2 (1996), 241-280.

[21] J. RoE, Lectures on Coarse Geometry, Univ. Lecture Ser. 31, Amer. Math. Soc., Providence, R. I., 2003.

[22] J. RoE, Band-dominated Fredholm operators on discrete groups, Integral Equations Oper. Theory, 51, 3 (2005), 411-416.

[23] B. YA. ShteInBerg, Compactification of locally compact groups and Fredholmness of convolution operators with coefficients in factor groups, Tr. St-Peterbg. Mat. Obshch., 6(1998), 242-260 (Russian). 